\title{
LOS ESTUDIOS DE SEGUIMIENTO DE LA POBLACIÓN GRADUADA COMO HERRAMIENTA PARA EL CAMBIO Y LA INNOVACIÓN EN EL CURRÍCULO UNIVERSITARIO: LA EXPERIENCIA DE LA ESCUELA DE ADMINISTRACIÓN EDUCATIVA DE LA UNIVERSIDAD DE COSTA RICA
}

\author{
Flora Eugenia Salas Madriz \\ Docente de la Escuela de Administración Educativa \\ de la Universidad de Costa Rica \\ San José, Costa Rica
}

Recibido 03-X-2006

Resumen: En este artículo se presentan resultados parciales para el caso de la Licenciatura en Administración Educativa del proyecto de investigación No. 241-A3-066, titulado Estudio de seguimiento de la población graduada de la Licenciatura en Ciencias de la Educación con énfasis en Administración Educativa y en Administración de programas de Educación no Formal (2004). Se exponen algunos aspectos del desarrollo histórico-social de las profesiones modernas, un marco de referencia general de los estudios de graduados y del perfil del egresado, así como una reseña de la formación en el campo de la Administración Educativa en la Universidad de Costa Rica.

Se muestran algunos resultados y el análisis de la información que se obtuvo a través de la técnica del grupo de discusión para conocer la opinión de los graduados del 2003 de la provincia de Alajuela, con la formación recibida a lo largo de toda la carrera. También, se exponen algunas conclusiones generales sobre ventajas y limitaciones de los estudios de seguimiento de la población graduada para el cambio y la innovación del currículo universitario, y se comenta la experiencia del empleo de esta herramienta en la Escuela de Administración Educativa para tal propósito.

Palabras clave: Estudios de seguimiento de la población graduada, perfil del graduado, perfil profesional, formación profesional, cambio e innovación en el currículo universitario, cambio e innovación curricular en Administración Educativa.

\section{Introducción}

En el 2003, para revisar los planes de estudio que se imparten en la Escuela de Administración Educativa de la Universidad de Costa Rica (UCR), fue preciso conocer la percepción general de los graduados de la carrera de Licenciatura en Administración Educativa. Para ello se inscribió un proyecto de investigación, cuyo objetivo general fue identificar las características del ejercicio profesional y la inserción laboral de la población graduada durante el período 1998-2002. Los objetivos específicos del estudio fueron los siguientes:

1. Obtener una base de datos con la información académica y laboral sistematizada de la población graduada en el período 1998-2002.

2. Determinar el perfil de ingreso y de salida del estudiantado.

3. Identificar el grado de satisfacción con la formación recibida de personas graduadas en el período en estudio.

4. Obtener información para actualizar el plan de estudios de la Licenciatura 


\begin{abstract}
This paper presents partial results of the research project titled Graduate Tracer Studies for the Education Science with Emphasis on Education Administration, which was carried out between 2003 and 2005. A general theoretical framework of this type of studies, relevant aspects about the historical-social development of modern careers, graduate profile, and the career in Education Administration at the University of Costa Rica. Partial results are presented on the opinion about the career of the graduates of the 2002 in Alajuela, in addition to the information analysis obtained through the focal group technique. Some conclusions are highlighted in relation to the advantages and limitations of the tracer studies for change and innovation of the college curriculum in general, and particularly, about the experience in using this tool in the Education Administration.
\end{abstract}

Key words: Graduate tracer studies, graduate profile, professional profile, professional development, change and innovation in the college curricula, change and innovation in the Education Administration curriculum. en Administración Educativa y para desarrollar programas de educación continua para estos profesionales.

Posteriormente, se hace una introducción al origen y desarrollo histórico-social de las profesiones modernas, a los estudios de seguimiento de la población graduada, a los perfiles profesional y académico, y una reseña de la formación de profesionales en el campo de la Administración Educativa en la UCR, a partir de 1990. Finalmente, se presentan resultados parciales del estudio para el caso de la Licenciatura en Ciencias de la Educación con énfasis en Administración Educativa, ventajas y limitaciones de los estudios de seguimiento de la población graduada en procesos de cambio e innovación curricular, y algunas reflexiones sobre la aplicación de esta metodología en la Escuela de Administración Educativa.

\section{El desarrollo histórico-social de las profesiones modernas}

Las profesiones son un fenómeno propio de la Era Industrial y se han desarrollado para satisfacer los requerimientos y necesidades del sistema productivo. Si bien cada profesión tiene su propio desarrollo estructural, todas responden a “...circunstancias propias del orden institucional prevaleciente" (Pacheco \& Días, 1997, p. 33). Dado que las profesiones reflejan las coyunturas históricas por las que atraviesa la sociedad, los cambios y grado de desarrollo del conocimiento y la tecnología tienen un impacto sobre ellas. De igual modo, las tendencias económicas dominantes y la lógica política que le subyace privilegian o desestiman actividades profesionales, razón por la cual el estudiantado se inclina por escoger carreras que gocen de mayor aceptación y reconocimiento social, debido a que son las que reciben mayor compensación salarial. Desde esta perspectiva, se reconoce a la persona profesional 
...como una figura historizada, [que] representa el producto social más acabado y condensa a todo un conjunto de condiciones, procesos y prácticas sociales articuladas en general, por un lado, por la demanda social, y, por otro, por un campo de conocimiento estructurado a partir de la demanda. El campo y el profesional que lo representa están sujetos al influjo de los diversos factores aquí señalados: de coyuntura, de posiciones y fuerzas sociales, de reorientación en cuanto al tipo de demanda a la que históricamente se enfrenta el campo, y de formas de especialización producidas en el interior del campo. (Pacheco \& Días, 1997, p. 35)

Existe un vínculo entre el grado de desarrollo del conocimiento, las tecnologías asociadas a ese desarrollo y las demandas e intereses sociales prevalecientes, por lo que el perfil de la persona que egresa de un plan de estudios queda determinado por estos factores, como se verá a continuación.

\subsection{El perfil del egresado}

Los programas de docencia se manifiestan en tres niveles: el perfil del egresado, el plan de estudios y los procesos educativos. Todos estos aspectos inciden en el cumplimiento de las condiciones para que la educación superior sea de calidad, pertinente y equitativa. Por perfil del egresado se entiende la formación general que ofrecen las instituciones de educación superior. Se sustenta en una propuesta curricular que refleja los fines y principios de la universidad y tiene que ver con el desempeño que se espera en la práctica de la disciplina y en el ejercicio profesional (Glazman, 2001), por esta razón,

Representa la síntesis de los resultados formativos más importantes de la institución educativa. Está constituido por las actitudes, valores, actividades, procedimientos, características, funciones y papeles requeridos por la práctica profesional y social. En términos generales, el perfil del egresado es una representación del sujeto que las instituciones de educación superior buscan formar. Es el resultado de la forma en que dichas instituciones conciben los ámbitos social, profesional y académico y constituye una importante fuente para formular el plan de estudios, ya que define los marcos filosófico, educativo y cultural de la formación. El perfil debe, por lo tanto, prever que el egresado al adquirir los conocimientos y habilidades en las prácticas de determinadas áreas, cuente con los elementos que le permitan resolver los distintos problemas de un campo específico de actividad. (Glazman, 2001, p. 124)

El perfil del egresado expresa las distintas dimensiones de la formación universitaria, porque involucra el proyecto académico de la carrera, los campos disciplinarios, científicos y técnicos asociados a la disciplina, los intereses y necesidades académicas del estudiantado y del personal docente, los sectores laborales en que tiene lugar el desempeño profesional, la cultura institucional universitaria, las demanda social de la profesión, y, finalmente, las tendencias de desarrollo nacional y local (Glazman, 2001).

Dadas sus características, el perfil del egresado constituye un "...referente específico de las condiciones concretas y particulares de la práctica social y profesional" (Glazman, 2001, p. 127). Por esta razón, para definir el perfil del egresado se debe tomar en cuenta que:

a. Los aspectos formativos en la educación superior se relacionan con la formación de una conciencia social.

b. La práctica profesional está sujeta a una amplitud de variaciones en función de los cambios científicos y tecnológicos en cada área, de las modificaciones en las demandas de los sectores de la producción, del ámbito geográfico de la práctica profesional, de las empresas que la desarrollan y de los cambios axiológicos y sociales relacionados con la profesión. (Glazman, 2001, p. 127)

\subsection{El perfil profesional y el perfil académico}

El perfil del egresado se conforma, como se mencionó, de los conocimientos, habilidades, actitudes y valores necesarios para el adecuado desempeño profesional; esto es, de todos los requisitos cognoscitivos, actitudinales y éticos para que haya un apropiado desempeño en relación con las tareas, funciones, actividades y acciones que 
debe realizar el graduado en un momento histórico particular. El perfil del egresado tiene dos componentes: el perfil profesional $y$ el perfil académico. Por medio del perfil profesional se proporcionan al estudiantado los conocimientos, capacidades, habilidades y actitudes necesarios para un satisfactorio desempeño práctico. A través del perfil académico se apoya una formación en la dimensión ética, social y política, con el fin de que el estudiantado incremente su conciencia crítica y consolide una actitud socialmente responsable. También, a través del perfil académico se propicia el desarrollo de destrezas cognoscitivas y socioafectivas que favorezcan el aprendizaje y el dominio de la comunicación oral y escrita, todos ellos indispensables para el adecuado ejercicio de la profesión.

\section{Los estudios de seguimiento de la población graduada}

Los estudios de seguimiento de la población graduada surgieron como respuesta a la necesidad de las instituciones de educación superior de ajustar sus programas y planes de estudio a las cambiantes y diversas demandas del mundo del trabajo en la economía globalizada. Debido a esto, cuando se hace una revisión de los planes de estudio de una carrera universitaria se debe empezar por conocer la realidad del ejercicio profesional correspondiente. Es así como, en términos generales, la población graduada de los programas universitarios y el sector empleador de esos profesionales son una fuente imprescindible de información.

Los estudios de seguimiento de la población graduada parten del supuesto de que la actualización de los planes de estudio universitarios no puede hacerse al margen de la realidad social y económica de cada momento histórico, ni del desarrollo interno y contextual de cada país. Debido a que la sociedad y la economía son dinámicas, las universidades tienen que desarrollar metodologías para entender e interpretar el contexto laboral, social y económico para que, en forma periódica, hagan los cambios pertinentes a sus planes de estudio y programas de educación continua. Esto es importante, máxime si se trata de instituciones de educación superior pública que tienen la obligación de hacer un adecuado uso de los recursos limitados de los que disponen para alcanzar niveles más elevados de eficiencia y eficacia en la formación profesional.

Hasta el momento, los estudios de seguimiento de la población graduada y la consulta a los sectores empleadores constituyen una forma de investigación empírica que ha demostrado su efectividad para evaluar los resultados de la formación profesional y de la capacitación que proveen las instituciones de educación superior (Schomburg, 2001). Desde esta perspectiva el Centro para la Investigación en Educación Superior y Trabajo de la Universidad de Kassel, en Alemania, desarrolla estudios con poblaciones de graduados diseñados para contribuir a la evaluación y actualización de planes de estudio en diversas universidades y países del mundo.

Desde 1978, los estudios de seguimiento (tracer studies) son un campo privilegiado de investigación en el Centro para la Investigación en Educación Superior y Trabajo de la Universidad de Kassel. La investigación que se ha hecho desde entonces hasta el presente incluye diversas temáticas como: la expansión universitaria y su relación con el trabajo, la reestructuración de programas de estudio, el análisis de la complejidad de los planes de estudio y su importancia para el desarrollo profesional, el reclutamiento de profesionales y, la conexión entre las calificaciones esperadas en el tiempo de entrada al mercado laboral, la calificación inicial, y la carrera profesional posterior de la población graduada (Schomburg, 2003).

La relación entre formación profesional y mundo del trabajo es, como se puede 
observar, el eje de los estudios de seguimiento de la población graduada, debido a que la subcalificación, la calificación equivocada o la sobrecalificación profesional genera inconsistencias entre la oferta y la demanda en el mercado laboral que afectan no sólo la capacidad productiva del país y de las personas, sino también la economía y la sociedad como un todo (Pfeiffer, 1993). Por esta razón, es fundamental mantener un grado aceptable de coherencia entre el perfil de salida de la población graduada y los requerimientos del mundo del trabajo.

\section{La formación de profesionales en Administración Educativa en la Universidad de Costa Rica}

En 1991, la Escuela de Administración Educativa inició un proceso que condujo a la reestructuración del plan de estudios vigente a la fecha ${ }^{1}$. Con tal propósito, en el segundo semestre de ese año se realizaron diversas actividades, cuyo fin fue crear las bases para la reflexión y planteamiento conceptuales de los nuevos planes de estudio, que incluyó una tesis de licenciatura en la que se hizo el primer estudio de seguimiento de la población graduada de esa Unidad Académica.

El proceso contó con varias etapas que incluyeron el trabajo en equipo del personal docente de la Sección de Administración Educativa y de la comisión de Currículo de la Escuela. Además, contó con la participación activa y comprometida de la mayoría del personal administrativo y académico de la Escuela.

En relación con el contexto y las tendencias nacionales y su impacto en la formación profesional se hizo referencia a la consolidación de un estilo de desarrollo económico y social nacional y mundial fundamentado en la ciencia y la tecnología. Ello implicó la necesidad de formar profesionales en el campo de la Administración Educativa con una sólida base científico-tecnológica, capacidad innovadora y pensamiento crítico, para que se ajustaran de manera eficiente y eficaz, con sentido de responsabilidad social, a los cambios vertiginosos que caracterizan nuestro tiempo.

En cuanto a la Administración Educativa en el contexto de la modernización, se señaló que la educación, por ser un bien en sí mismo y repercutir de manera directa en la capacidad productiva y personal, debe garantizar un alto rendimiento de las inversiones personales y sociales. Por ello, es necesario que sea de calidad y para el logro de tal propósito la Administración Educativa es determinante, pues su intención es utilizar los recursos humanos, financieros y comunitarios de manera que se logren las metas educativas con un alto grado de eficiencia y calidad.

Entre los factores a administrar por profesionales de la Administración Educativa están los recursos humanos que en educación juegan un papel aún más importante que en otros sectores económicos y de servicios. El personal docente y el estudiantado constituyen el eje del acto educativo, razón por la cual quien ejerce el cargo de la administración de la educación debe contar con las habilidades y destrezas de comunicación, liderazgo y negociación necesarios para, en un sano clima organizacional, lograr la más alta motivación y, en consecuencia, el mejor desempeño posible de docentes y estudiantes.

Como otro elemento fundamental está la comunidad en la cual se inserta la institución educativa. Esta, al igual que los docentes y estudiantes, debe formar parte de las prioridades de quien administra la educación para que tenga la sensibilidad y el conocimiento suficiente de su entorno y procure reducir las desventajas socioculturales que impactan el rendimiento académico. Con base en lo anterior, la formación de profesionales en administración de la educación debe fundamentarse en una profunda base pedagógica y en una formación administrativa de calidad. 
Entre las bases epistemológicas de la formación de profesionales en el campo, se señaló que la Administración Educativa se construye como disciplina a partir de los aportes de la Pedagogía y la Administración. Por ello, se deben considerar ambas disciplinas como el eje articulador de la formación. Sin embargo, para entonces todavía se pensaba que la Administración Educativa no había alcanzado la madurez teórico-práctica necesaria para considerarla una disciplina científica propiamente dicha. Por esta razón, en aquel momento se concluyó que estaba en proceso de "construcción", pero que tenía muchas posibilidades de constituirse como tal en el corto plazo.

Para el nuevo plan de estudios de 1993 se utilizó el enfoque sociocrítico de la Administración Educativa para dar sustento epistemológico y teórico a la carrera, pues en este enfoque se parte del supuesto de que la realidad es compleja y que en el tejido social existen estructuras de poder y conflictos sociales. Además, en el nivel pedagógico se partió de una posición epistemológica constructivista que considera que los individuos se configuran como tales, a través del contacto activo con la realidad que les permite realizar procesos de construcción y reconstrucción permanente de lo psicológico, del entorno y del entramado sociocultural (Escuela de Administración Educativa. Planes de Estudio Licenciatura en ciencias de la Educación con énfasis en Administración Educativa y Administración de Programas de Educación no Formal, 1993).

Luego de esas reflexiones se eligió el enfoque sociocrítico de la disciplina como base de la formación en la Escuela de Administración Educativa, pues permite superar las limitaciones propias de las perspectivas interpretativas y tradicionales. Al respecto, se concluyó que el enfoque interpretativo presenta problemas, debido a que carece de una perspectiva crítica. Por su parte, el enfoque tradicional o positivista se caracteriza por una pretensión de cientificidad y neutralidad ética poco coherentes con la realidad educativa. El enfoque sociocrítico permitiría superar las visiones acríticas y tecnocráticas de la Administración Educativa que habían prevalecido hasta entonces.

Como base del perfil profesional en el campo de la Administración Educativa se propuso las áreas pedagógica y administrativa. El área pedagógica da los conocimientos necesarios para la comprensión del proceso de enseñanza y aprendizaje, que constituye la razón de ser de la educación. Los conocimientos de la teoría de la administración proporcionan el desarrollo de habilidades específicas del área, imprescindibles para un adecuado desempeño en la gestión de organizaciones educativas.

Respecto de la formación de profesionales en Administración Educativa, se señaló que Walton (citado en: Escuela de Administración Educativa. Planes de Estudio Licenciatura en ciencias de la Educación, 1993) propone tres modelos que se caracterizan porque:

1. La persona que se gradúa en Administración Educativa es concebida como especialista en educación. Este es el modelo que ha predominado en el país.

2. La función administrativa es la misma, independientemente del tipo de organización, por lo que se hace énfasis en las técnicas y habilidades ejecutivas y empresariales.

3. Propone que las organizaciones educativas son complejas, por lo que quienes administran centros educativos deben ser especialistas en el manejo de la información y en la toma de decisiones.

Debido a que en Costa Rica predomina la concepción de profesionales en Administración Educativa como administradores del currículo, se cae dentro del 
modelo que les considera especialistas en educación. En el plan de estudios propuesto en 1993 se:

...asume que un administrador educativo debe tener una clara formación pedagógica y experiencia docente, asimismo, una sólida formación administrativa. $\mathrm{Su}$ función consiste en contribuir mediante su gestión al logro de metas institucionales que conlleven la obtención de una educación de calidad. Esta se manifestará en el desarrollo integral de los estudiantes, tanto en el dominio de conocimientos básicos, como en la adquisición de habilidades, destrezas y actitudes fundamentales en el marco de una educación para la democracia participativa. (Escuela de Administración Educativa. Planes de Estudio Licenciatura en Ciencias de la Educación con énfasis en Administración Educativa y Administración de Programas de Educación no Formal, 1993, pp. 16-17)

Como resultado de estas reflexiones se propuso un perfil profesional para la Licenciatura en Administración Educativa, que debía contener las características generales necesarias en lo que respecta a conocimientos, cualidades personales, habilidades, actitudes y valores. El mismo fue hecho con base en las características del desempeño en la realidad, y los fundamentos epistemológicos y teóricos de la disciplina.

Para obtener el perfil real se tomó como base la investigación para obtener el grado de Licenciatura en Administración Educativa en la que se hizo el primer estudio de seguimiento de la población graduada de la Escuela, en el que se llegó a las siguientes conclusiones:

1. El 50\% de los graduados no labora en cargos administrativos.

2. El proceso de selección y nombramiento de directores de centros educativos privilegia la experiencia sobre la formación en el campo.

3. Los graduados que se desempeñan en cargos administrativos dedican la mayor parte de su tiempo a:

a. mantenimiento de la planta física,

b. búsqueda de recursos materiales y económicos,

c. atención al público,

d. trámite de correspondencia.
Por esta razón, no pueden atender como corresponde aspectos fundamentales como la administración dela educación. (Escuela de Administración Educativa. Planes de Estudio Licenciatura en Ciencias de la Educación con énfasis en Administración Educativa y Administración de Programas de Educación no Formal, 1993, pp. 17).

En cuanto a la formación recibida en la Escuela de Administración Educativa, el estudio señaló que:

1. El plan de estudios no conduce a una formación tal que permita el manejo de modelos curriculares diversos, la adaptación del currículo a la comunidad, ni la administración de personal de modo que se obtengan los mejores logros en el proceso de enseñanza-aprendizaje.

2. Es necesario enriquecer la formación con elementos de informática, legislación educativa, teorías de aprendizaje, aspectos didácticos, procedimientos de evaluación institucional y regional, destrezas en el manejo de personal, elaboración de planes y programas, y tareas cotidianas del administrador (archivo, presupuesto, confección de cuadros y otros similares).

3. Los cursos Educación Comparada y Seminario de Temas Varios en Administración Educativa aportan poco para el desempeño.

4. El Plan de Estudios enfatiza aspectos teóricos desactualizados en algunos campos y deja de lado los aspectos prácticos y las innovaciones en el área de la administración.

5. Las metodologías y los recursos didácticos que se utilizan para formar a los administradores educativos son de corte academicista y priorizan los aspectos cognoscitivos. No conducen al desarrollo de la actitud crítica e investigativa de los estudiantes, ni favorecen el desarrollo de destrezas y habilidades intelectuales o prácticas.

6. El Plan de Estudios vigente ha permanecido sin cambios sustanciales durante varios años, en tanto la administración se ha desarrollado tecnológicamente en forma acelerada para dar respuesta a los constantes cambios del mundo actual. Por su parte, la Pedagogía en América Latina ha planteado modelos novedosos para la solución de los problemas educativos de la región. (Escuela de Administración Educativa. Planes de Estudio Licenciatura en Ciencias de la Educación con énfasis en Administración Educativa y Administración de Programas de Educación no Formal, 1993, pp. 18)

El perfil profesional se pensó para que la población graduada pudiera 
desempeñarse en el Ministerio de Educación Pública en los niveles central (formulación y gestión de políticas educativas), intermedio (Administración de sedes regionales y subregionales) e institucional (dirección de centros educativos). Las funciones que podrían desempeñar las personas graduadas son las siguientes:

1. Planear, organizar, coordinar, dirigir, supervisar y evaluar el proceso educativo que se desarrolla en la institución.

2. Organizar el trabajo y los recursos humanos y materiales de modo que se logren los objetivos educativos e institucionales.

3. Generar una relación dialéctica entre la institución y la comunidad.

4. Propiciar la readecuación curricular de acuerdo con las necesidades y demandas sociales y culturales.

5. Procurar que se realice la realimentación de los procesos educativos e institucionales, de acuerdo con los avances científico-tecnológicos, tanto desde el punto de vista pedagógico, como administrativo.

6. Desarrollar programas para el buen mantenimiento de los recursos físicos y materiales de la institución.

7. Ejercer el liderazgo y tomar las decisiones adecuadas para propiciar metas de calidad educativa en la institución.

8. Coordinar, supervisar y evaluar programas adecuados para la atención de los problemas y el desarrollo de los estudiantes.

9. Atender oportunamente los conflictos sustantivos de la institución.

10. Establecer mecanismos adecuados de comunicación.

11. Fomentar buenas relaciones interpersonales y grupales.

12. Promover el mejoramiento profesional continuo de los educadores y otros funcionarios de la institución.

13. Consultar a los especialistas y asesorarse adecuadamente cuando la situación así lo requiera.

14. Elaborar y aplicar procedimientos normativos que coadyuven y orienten la labor de la institución.

15. Establecer mecanismos de evaluación institucional.

16. Organizar actividades cívicas y culturales que propicien el desarrollo integral de los educandos y el rescate de los valores de la nacionalidad costarricense.

17. Conocer y aplicar las políticas educativas nacionales y regionales en la planificación de los procesos curriculares institucionales.
18. Participar en reuniones, seminarios, juntas y otras actividades en la institución o la comunidad, con el fin de contribuir a los objetivos que se propone la institución que dirige.

19. Representar el cargo que desempeña ante organismos públicos y privados.

20. Elaborar e interpretar informes, instructivos u otros documentos que el cargo le exige. (Escuela de Administración Educativa. Planes de Estudio Licenciatura en ciencias de la Educación, 1993, pp. 19-20).

En cuanto a las características y condiciones personales deseables para los(as) graduados(as) se señalaron las siguientes:

- Iniciativa

- Confianza y seguridad en sí mismo.

- Sensibilidad y responsabilidad social y profesional.

- $\quad$ Respeto por el ser humano y por la vida.

- Motivación para la búsqueda y logro de metas.

- Tendencia al liderazgo.

- Capacidad para conducir grupos.

- Habilidad en el manejo de relaciones interpersonales.

- Capacidad de organización.

- Capacidad para enfrentar conflictos.

- Tendencia a la actualización profesional.

- Disposición positiva ante el cambio y la innovación.

- Comportamiento ético en el desempeño profesional.

- Capacidad para tomar decisiones.

- Adecuada autoestima.

- Interés por la realidad nacional y por la construcción de la democracia.

- Facilidad para comunicarse.

- Espíritu investigativo.

- $\quad$ Actitud científica.

- Interés por el desarrollo de quienes lo rodean.

- Clara conciencia sociopolítica. (Escuela de Administración Educativa. Planes de Estudio Licenciatura en ciencias de la Educación, 1993, pp. 20-21)

Para cumplir con el perfil profesional propuesto, se organizó el plan de estudios a partir de tres áreas: la pedagógica, la administrativa y la investigación. Los objetivos del plan de estudios de 1993, aún vigente, son los siguientes:

1. Formar profesionales capaces de adminis trar centros o procesos educativos de modo 
que se contribuya al desarrollo integral del educando y de la comunidad circundante y nacional.

2. Ofrecer al administrador educativo una sólida formación en los campos de la teoría y de la técnica administrativa, de modo que sea capaz de ejercer una gestión administrativa que coadyuve al logro de las metas educativas nacionales e institucionales.

3. Coadyuvar mediante un adecuado proceso de formación al desarrollo de la capacidad de liderazgo de los futuros administradores educativos de modo que sean promotores del cambio y la innovación educativa en las instituciones que dirijan.
4. Formar profesionales capaces de analizar las políticas educativas nacionales con espíritu crítico, de aportar sugerencias y de llevarlas a la práctica mediante adecuados procesos de planificación y organización institucional.

5. Ofrecer oportunidades metodológicas y prácticas para que el futuro administrador educativo ejercite su potencial creativo como conductor de procesos educativos y administrativos. (Escuela de Administración Educativa. Planes de Estudio Licenciatura en ciencias de la Educación, 1993, pp. 26-27).

El plan de estudios vigente de la carrera es el siguiente:

Cuadro 1

\begin{tabular}{|c|c|c|}
\hline \multicolumn{3}{|c|}{ Plan de Estudios vigente Licenciatura en Administración Educativa } \\
\hline \multicolumn{3}{|c|}{ PRIMER SEMESTRE } \\
\hline No. del curso & Nombre del curso & Créditos \\
\hline EA-0388 & Fundamentos y desarrollo de la teoría administrativa & 3 \\
\hline EA-0390 & Sociología de la Educación & 3 \\
\hline EA-0396 & Principios de Investigación & 3 \\
\hline EA-0399 & Epistemología Educativa & 3 \\
\hline \multicolumn{2}{|l|}{ Total de créditos } & 12 \\
\hline \multicolumn{3}{|c|}{ SEGUNDO SEMESTRE } \\
\hline EA-0389 & Planificación y Desarrollo en la Administración Educativa & 3 \\
\hline EA-0394 & Tecnología educativa & 3 \\
\hline EA-0400 & Taller de Investigación & 3 \\
\hline EA-0421 & Comportamiento Organizacional en la Educación & 3 \\
\hline \multicolumn{2}{|l|}{ Total de créditos } & 12 \\
\hline
\end{tabular}


Cuadro 1 (Continuación)

\begin{tabular}{|l|l|c|}
\hline No. del curso & Nombre del curso & Créditos \\
\hline \multicolumn{2}{|c|}{ TERCER SEMESTRE } \\
\hline EA-0391 & Administración de los Recursos Humanos en el Sistema Educativo & 3 \\
\hline EA-0393 & Supervisión y Evaluación en Administración Educativa & 3 \\
\hline EA-0180 & Gestión de Procesos Legales & 3 \\
\hline Total de créditos & & 9 \\
\hline Total de créditos de la carrera & 33 \\
\hline
\end{tabular}

Fuente: Escuela de Administración Educativa. Plan de Estudios de la Licenciatura en Ciencias de la Educación con énfasis en Administración Educativa.

La Escuela de Administración Educativa ofrece como modalidades de graduación en este Plan de Estudios: Tesis de graduación, Seminario de graduación, Proyecto de graduación y Práctica dirigida. El mismo se ha modificado parcialmente a la fecha, ya que se eliminaron dos talleres y se cambió el curso EA-0401 Sistemas de Información para Administradores Educativos, por un curso de legislación. Actualmente se está en un proceso de revisión del plan de estudios para incorporar las sugerencias de la población graduada, así como los hallazgos hechos en otras investigaciones con fines de acreditación de la carrera.

\section{Metodología del estudio}

Como parte de la metodología del estudio, se utilizó el grupo de discusión para conocer la opinión de algunas personas graduadas en relación con la formación profesional recibida. El grupo de discusión se suele clasificar como una forma de entrevista grupal que pueden ser de distinto tipo: grupos focalizados, brainstorming o lluvia de ideas, grupos nominales y Delphi (consulta a expertos) y entrevistas grupales de campo, naturales y formales. Se define como una técnica típica para explorar temas o aspectos de interés para la investigación, y pretenden que las personas que brindan información den sus puntos de vista, apreciaciones y valoraciones sobre los temas en estudio. La técnica de grupos focalizados o de discusión ocupa un lugar privilegiado para la obtención de información cualitativa en las ciencias sociales.

Para efectos del estudio, se convocó a un grupo de personas graduadas de la Licenciatura en Administración Educativa de la generación 2000-2002, de la provincia de Alajuela. Acudieron voluntariamente ocho estudiantes. El grupo de discusión tuvo como metodología plantear siete preguntas para conocer su opinión respecto de:

- $\quad$ El plan de estudios y los cursos de la carrera;

- La calidad del personal docente.

- La experiencia con el trabajo final de graduación.

- Los servicios administrativos.

- La proyección de la misma en la comunidad de especialistas. 
- La pertinencia de la carrera, entendida como el grado de coherencia entre la formación recibida y los requerimientos reales del desempeño profesional.

Contaron con 30 minutos para responder a estas preguntas en forma individual. Posteriormente tuvieron una hora para discutir en grupo sus opiniones, a fin de llegar a una opinión de consenso respecto de los temas en cuestión. Finalmente, se eligió a una persona para exponer y comentar las conclusiones del grupo. A continuación se ofrecen los resultados del grupo de discusión.

\subsection{Resultados del grupo de discusión}

Las respuestas de los(as) estudiantes se presentan en cuadros que resumen la opinión del grupo sobre los temas en discusión. Posteriormente, se hace un análisis de la información obtenida.

\subsubsection{Opinión sobre el plan de estudios y los cursos de la carrera}

Las conclusiones del grupo se dividieron en carencias del plan de estudios y de los cursos, a fin de discriminar problemas generales de problemas específicos.

Señalan las personas graduadas que el plan de estudios se debe revisar, pues tiene vacíos importantes en el campo específico de la Administración Educativa, así como en áreas críticas como la tecnología, la legislación y los sistemas de información. Como se señaló anteriormente, las profesiones deben estar acordes con las demandas sociales para satisfacer las necesidades específicas del campo. De lo contrario, tanto en el nivel social como en el personal, estos desfases van en detrimento de la productividad y la calidad de vida. Esta situación también se manifiesta en el señalamiento de que la carrera está desvinculada de la realidad. Esta es una situación que debe corregirse si se quiere que estas profesiones sean competitivas.
Una opción para resolver las deficiencias mencionadas en la formación profesional es la creación de un programa de educación continua, que permita subsanar estas carencias y elevar la calidad del desempeño profesional. Un aspecto que se debe corregir es la debilidad en la formación en investigación, ya que profesionales en el campo de la Administración Educativa deben tener sólidas bases en esta área, pues su desempeño laboral dependerá en mucho de sus habilidades y conocimientos al respecto. La capacitación del personal docente de la Unidad Académica, así como la definición de políticas claras sobre investigación en la Escuela de Administración Educativa son una condición para resolver este problema.

\subsubsection{Opinión sobre la calidad del personal docente}

La respuesta grupal se dividió en aspectos negativos, aspectos positivos y sugerencias de las personas graduadas para mejorar los problemas detectados en relación con el personal docente.

Como en los señalamientos sobre el tema anterior, las personas graduadas mencionaron la necesidad de un plan de desarrollo del personal docente de la Escuela de Administración Educativa, acompañado de la evaluación del desempeño, la formación y realimentación continua. La renovación en las prácticas y métodos de enseñanza del profesorado es indispensable para elevar la calidad de la formación que ofrece la Escuela. Si bien se advierten problemas con algunos docentes, también se menciona que, en términos generales, el profesorado reúne condiciones para una formación profesional de excelencia. Esto es evidencia de la necesidad de hacer un plan de evaluación permanente de la práctica docente, que incluya la opinión del estudiantado y la revisión de la realidad y condiciones de la docencia en la Escuela de Administración Educativa.

En educación superior es común que el profesorado no tenga formación 


\section{Cuadro 2}

Opinión sobre el Plan de estudios y de cursos de la carrera

\begin{tabular}{|c|c|}
\hline Carencias & Respuestas \\
\hline Del Plan de estudios & $\begin{array}{l}\text { 1. Debe ajustarse a la realidad costarricense. } \\
\text { 2. Se deben conectar los cursos con la realidad. } \\
\text { 3. El plan de estudios está desarticulado debido a que: } \\
\text { a. No hay integración entre el programa y los contenidos. } \\
\text { b. Algunos contenidos son obsoletos. } \\
\text { c. Se carece de contenidos que son fundamentales en la formación de } \\
\text { un(a) administrador(a) educativo(a), como los siguientes: } \\
\text { i. Legislación educativa. } \\
\text { ii. Estadística aplicada al campo de la administración educativa. } \\
\text { iii. Presupuesto, planillas, horarios. } \\
\text { iv. Planificación. } \\
\text { v. Sistemas de información. } \\
\text { vi. Tecnología aplicada a la Administración Educativa. } \\
\text { El tiempo para graduarse es excesivo por lo que el trabajo de investiga- } \\
\text { ción debe comenzar en el segundo semestre y no dejarse para el final } \\
\text { de la carrera. } \\
\text { El plan de estudios repite algunos contenidos del bachillerato y hay } \\
\text { incongruencias entre los contenidos recibidos en la carrera base y los } \\
\text { de la Licenciatura en Administración Educativa. }\end{array}$ \\
\hline De los cursos & $\begin{array}{l}\text { 1. Hay cursos obsoletos, en los que no se utilizan metodologías innovado- } \\
\text { ras ni las nuevas tecnologías. } \\
\text { 2. Algunos talleres son obsoletos, ya que no hay relación entre el progra- } \\
\text { ma y los temas que se aborda en los cursos. } \\
\text { 3. El Trabajo Comunal Universitario debe ser funcional y práctico. } \\
\text { 4. Los cursos de investigación utilizan bibliografía vieja, de más de quin- } \\
\text { ce años atrás, fueron poco claros en cuanto a metodología. } \\
\text { 5. En el curso de investigación no se logró una perspectiva de proceso, } \\
\text { fue muy confuso y contribuyó poco o nada al abordaje del proyecto de } \\
\text { graduación. } \\
\text { En los cursos no hay criterios claros de evaluación, lo que confunde } \\
\text { a los(as) estudiantes y no les permite tener criterios claros sobre sus } \\
\text { progresos, limitaciones y áreas débiles. }\end{array}$ \\
\hline
\end{tabular}

pedagógica y que no siga un plan coherente de evaluación del desempeño y de formación continua. Dadas las condiciones actuales de desarrollo del conocimiento en general y de la disciplina en particular, así como a los cambios en la enseñanza y el aprendizaje es indispensable tomar en cuenta estos aspectos para atender deficiencias y limitaciones oportunamente. Lo más importante de un plan de estudios es su implementación y, por ende, las previsiones que se tomen para garantizar que será coherente con lo previsto en el nivel de diseño. Si no se toma en cuenta un plan de inducción, evaluación y desarrollo del personal académico difícilmente se alcanzará el éxito esperado.

\subsubsection{Opinión sobre la experiencia con el trabajo final de graduación}

Para sistematizar la respuesta sobre este tema se organizó la información en dos 
Cuadro 3

Opinión sobre el personal docente de la Escuela de Administración Educativa

\begin{tabular}{|c|c|}
\hline Opinión & Respuestas \\
\hline Aspectos negativos & $\begin{array}{l}\text { 1. Hay docentes inconsistentes y desinteresados. } \\
\text { 2. Algunos profesores o profesoras tienen mucho trabajo en otras instituciones } \\
\text { y no pueden dedicar tiempo al estudiante ni a preparar adecuadamente el } \\
\text { curso. } \\
\text { 3. Algunos docentes tienen problemas con la administración del curso: } \\
\text { a. Mala planificación. } \\
\text { b. Evaluación poco clara (no hay retroalimentación al estudiante). } \\
\text { c. La evaluación es subjetiva en algunos casos, lo que desmotiva al estu- } \\
\text { diante. }\end{array}$ \\
\hline Aspectos positivos & $\begin{array}{l}\text { 1. La mayoría del personal docente da un buen trato al estudiante. } \\
\text { 2. Muchos docentes promueven el pensamiento crítico y reflexivo. } \\
\text { 3. La mayoría son flexibles y comprensivos. } \\
\text { 4. Muestran dominio y conocimiento de los temas y contenidos de los cursos. } \\
\text { 5. Algunos docentes son muy comprometidos y responsables. }\end{array}$ \\
\hline Sugerencias & $\begin{array}{l}\text { 1. Se debe capacitar al profesorado. } \\
\text { 2. Se debe establecer un balance entre la teoría y la práctica, ya que algunos } \\
\text { docentes son excesivamente teóricos y no logran establecer una relación } \\
\text { entre la teoría y la realidad. } \\
\text { 3. Se debe escoger mejor y evaluar al profesorado. } \\
\text { 4. Se deben hacer procesos de supervisión que permitan que el curso se man- } \\
\text { tenga en un nivel aceptable de calidad. }\end{array}$ \\
\hline
\end{tabular}

\section{Cuadro 4}

Experiencia con el trabajo final de graduación

\section{Problemas}

- Hay poca relación entre la formación recibida y el trabajo de graduación.

- Poca pertinencia de los cursos de investigación.

- Problemas con el diseño de investigación.

- Es necesario contar con mejor formación en estadística y en técnicas de investigación.

- La relación con docentes asesores (Dirección del proyecto de graduación y docentes lectores) debe cambiar. Los lectores no participan en el proceso.

- En algunas ocasiones hay poca dedicación por parte del director(a).

\section{Recomendaciones}

- Dar más orientación al estudiantado.

- Debe haber un mayor involucramiento del profesorado que tiene a su cargo la dirección de proyectos de graduación. 
partes: problemas en relación con el trabajo final de graduación y recomendaciones para su eventual solución.

Para el estudiantado es necesario iniciar el proceso de investigación con el comité asesor lo antes posible, para elevar la calidad y pertinencia de la orientación que reciben conforme desarrollan el proyecto de graduación. Señalan que quienes trabajan como lectores deben desempeñar un papel más activo en el proceso de la investigación, pues ello enriquecería la experiencia. Consideran que conviene una mayor claridad en cuanto a las políticas de la Escuela en materia de investigación, ya que se dan incoherencias entre la posición de un profesor y otro. La labor quien dirige el proyecto tiene que ser más efectiva y comprometida, porque piensan que recibieron poco apoyo, razón por la cual tardaron más en finalizar sus proyectos.

La investigación implica conocimientos diversos que rebasan con mucho los específicamente disciplinares. Para investigar se requieren amplios conocimientos epistemológicos, teóricos, metodológicos y técnicos. Si se quiere, la investigación constituye ella misma una especialidad que pocos profesionales logran dominar. Por esto, uno de los campos más débiles en la formación profesional lo constituye el profesorado mismo, pues, como señalan las personas que participaron en el grupo de discusión, hay evidencias claras de confusión y dificultades al respecto en la planta docente. Esta es un área que se debe fortalecer primero en el personal académico $\mathrm{y}$, posteriormente a través de la docencia en el estudiantado. No es posible ser un buen docente si no se es también un buen investigador. No se aprende investigación con un profesorado que no investiga.

Las universidades completas, que realizan investigación, docencia y acción social, son las que mayores posibilidades tienen para resolver los problemas propios de la enseñanza de la investigación. Si no lo hacen, ello implica que se han perdido las líneas de acción que unen las actividades fundamentales de la enseñanza universitaria. De nuevo, la atención a las características, perfil y necesidades del profesorado parece ser uno de los factores clave para la solución de estos problemas.

\subsubsection{Opinión sobre los servicios administrativos}

Es necesario mejorar la atención al estudiantado, debido a que en ocasiones se sintieron maltratados y confundidos. Opinan que en distintos momentos no recibieron la información con el tiempo necesario, razón por la cual perdieron beneficios o vieron lesionados sus intereses. Con base en estos señalamientos, es preciso que la Escuela de Administración Educativa revise sus políticas de atención al estudiante

Cuadro 5

Servicios administrativos

Respuestas

1. Los trámites son deficientes.

2. El personal de la secretaría no trata bien a los(as) estudiantes.

3. Hay desinformación y muchas veces se pierden beneficios universitarios porque no se avisa a tiempo (becas, por ejemplo).

4. No hay lineamientos claros en cuanto a investigación.

5. Los trámites en la universidad son engorrosos.

6. Hay exceso de burocracia. No se piensa en la comodidad y facilidad del (la) estudiante. 
y capacite a docentes-administrativos y al personal administrativo para ofrecer un servicio orientado al cliente, donde se busque la comodidad y satisfacción de las necesidades del estudiantado.

Esta situación se agrava en promociones desconcentradas que reciben con mayor dificultad la información y debido a que muchos trámites sólo se pueden hacer en las oficinas centrales en la Sede Rodrigo Facio. Por esta razón, eventualmente tienen que viajar a San José a realizar trámites confusos que involucran desde la matrícula hasta el pago de recibos. Es fundamental que en la Escuela de Administración Educativa las labores de coordinación de la carrera incluyan un sólido plan de simplificación de trámites administrativos, donde se garantice que el estudiantado tenga la menor cantidad de problemas y limitaciones posibles. Este podría incluir folletos de información general que se entreguen al iniciar cada ciclo lectivo. También, las tecnologías de la información y la comunicación (TIC) ofrecen en la actualidad muchas posibilidades para informar al estudiantado a través del correo electrónico, en particular si se trata de promociones desconcentradas, con poco o difícil acceso a la Sede Rodrigo Facio. El empleo combinado de medios de comunicación puede ser una alternativa para apoyar el trabajo de información general que deben asumir las personas que tienen a su cargo la coordinación y administración de la carrera.

\subsubsection{Opinión sobre la pertinencia de la carrera}

Estas respuestas señalan que hay problemas en cuanto al diseño del plan de estudios y, en consecuencia, de los cursos.

Cuadro 6

Pertinencia de la carrera

\section{Respuestas}

1. Hay cursos que se deben revisar, ya que están obsoletos. Particularmente el de Sistemas de Información.

2. Los cursos son excesivamente teóricos, por lo que se debe hacer un esfuerzo por equilibrar teoría y práctica.

3. Hay poca relación de los cursos con la realidad, por lo que su pertinencia es baja.

La vinculación de la actividad formativa con la realidad y las demandas del sector empleador pueden cerrar estas brechas. Se debe hacer un esfuerzo al interior de la Unidad Académica por revisar el material bibliográfico y las estrategias de evaluación, pues no responden a las necesidades actuales de la formación profesional.

Nuevamente, la formación del profesorado de la Unidad Académica, la vinculación de los cursos con la realidad de la profesión y el conocimiento del contexto nacional a través de la investigación, la docencia y la acción social son requisito para una formación adecuada en el campo de la Administración Educativa.

\subsubsection{Opinión sobre la proyección de la carrera en la comunidad de especialistas}

Con base en las conclusiones del grupo de discusión, la carrera no tiene impacto ni proyección en la comunidad de especialistas ni en el sector educativo nacional. Consideran indispensable que 


\section{Cuadro 7}

Proyección de la Escuela de Administración Educativa

\section{Respuestas}

1. No hay proyección de la carrera en el sector educativo.

2. Se debe crear un sistema de educación continua que permita el reciclaje profesional.

3. La investigación en Administración Educativa no se conoce, por lo que se debe mejorar y divulgar.

4. Se debe crear una publicación especializada en el campo de la Administración Educativa, que tenga un buen sistema de divulgación y distribución.

5. Debe haber mayor proyección a la comunidad.

haya más y mejor investigación, así como divulgación de sus resultados. Opinan que sería oportuno crear una revista especializada, que efectivamente llegue a quienes ejercen la profesión, por lo que debe ser de calidad y contar con un sistema de divulgación y distribución adecuado. Del mismo modo, creen que es necesario que la Escuela se proyecte más a la comunidad de especialistas, lo que podría lograrse a través de conferencias, seminarios o congresos nacionales e internacionales.

\subsubsection{Opinión sobre las políticas de contratación del Servicio Civil}

Las respuestas del grupo fueron diferenciadas en problemas y soluciones en relación con las políticas de contratación de profesionales del sector educación del Servicio Civil.

Es indispensable que la Escuela de Administración Educativa coordine acciones con el Ministerio de Educación Pública y el Servicio Civil, para mejorar la valoración profesional de la carrera.

Cuadro 8

Proyección de la Escuela de Administración Educativa

Problemas

1. Hay una calificación desigual de los profesionales y las profesionales en el campo de la Administración Educativa, ya que sólo se reconoce la Licenciatura si se ejerce el cargo.

2. Hay competencia desleal, porque no se discrimina entre universidades buenas y malas, por eso los(as) graduados(as) de universidades de prestigio como la Universidad de Costa Rica, compiten en igualdad de condiciones con universidades privadas de reconocida baja calidad.

3. El plan especial de Bachillerato en Administración Educativa no se reconoce como tal en el Servicio Civil.

4. Hay excesiva politiquería en el nombramiento y selección de personal en el área educativa, que también afecta a quienes se postulan a cargos de dirección.

\section{Soluciones}

1. Que la Escuela de Administración Educativa coordine con el Servicio Civil y haga una contrapropuesta del tratamiento actual de sus graduados(as).

2. Combatir y castigar la corrupción en el sistema educativo.

3. Evitar la intromisión de la política y que el MEP no se convierta en botín político.

4. El Servicio Civil debe diseñar estrategias para discriminar la calidad de los graduados y las graduadas.

5. Que las autoridades intervengan y controlen la formación de educadores(as) en general y de Administradores(as) de la educación en particular. 
Otro problema importante es la sobreoferta universitaria privada del grado de Licenciatura en Administración Educativa, pues al no haber ningún tipo de control de la calidad de la formación se están graduando profesionales que reconocen el Servicio Civil y el MEP como idóneos, sin que haya discriminación entre buenas y malas universidades. El estudiantado de universidades privadas se gradúa más rápido y obtienen títulos de postgrado con gran facilidad, razón por la cual hay una competencia desleal, en la medida en que unos tienen requisitos más altos que otros para obtener la misma acreditación profesional y laboral. Opinan que esta situación va en detrimento de la calidad profesional y, por ende, perjudica al sector educativo como un todo. Al respecto, creen que la Universidad de Costa Rica debe emprender acciones para corregir estas anomalías.

Lamentablemente, esta es una realidad que no sólo afecta a quienes se gradúan en Administración Educativa, sino al sector educativo en su totalidad. Ello muestra que la formación de profesionales en educación en el sector universitario privado se salió de control y exige medidas radicales e innovadoras. Entre ellas, se podría implementar un plan de certificación de idoneidad, en el que todos los profesionales sean sometidos a pruebas que garanticen su idoneidad para el ejercicio profesional. Evidentemente, estas soluciones rebasan las posibilidades de la Escuela de Administración Educativa, puesto que involucra políticas públicas de nivel nacional, que sean jurídicamente legitimadas e institucionalmente implementadas por el Servicio Civil y el MEP.

\section{Conclusiones}

Los estudios de seguimiento de la población graduada son una importante herramienta para identificar vacíos en la formación profesional en cualquier unidad académica. Las universidades no deben desestimar la implementación de este tipo de estudios para obtener información relevante de sus graduados en relación con su práctica profesional. Entre sus ventajas está que se puede contrastar la formación ofrecida con la realidad de la práctica profesional, así como registrar características del contexto laboral y determinar requerimientos emergentes en el sector empleador.

Otra de las ventajas de los estudios de seguimiento de la población graduada es que permiten identificar necesidades de formación y reciclaje profesional, que se perfila como una de las nuevas funciones de las universidades en el contexto de la Sociedad de la Información y el Conocimiento; esto es, determinar y satisfacer las necesidades de actualización de los profesionales que gradúa (UNESCO, 1998).

Una de las limitaciones de estos estudios es que son relativamente costosos, pues demandan tiempo de investigación y recursos materiales y financieros para ser exitosos. Además, no son suficientes por sí mismos, pues se requiere de información adicional del sector empleador para establecer características actuales y futuras de la práctica profesional. Ello supone costos adicionales que, al igual que en el estudio de seguimiento de la población graduada, requiere de recursos humanos y materiales para investigación. Si se cuenta con los recursos necesarios, se debe capacitar al personal de investigación en este tipo de estudios para que realicen procesos que culminen con la información necesaria para ajustar, cambiar e innovar el plan de estudios.

Una de las tendencias más importantes en la enseñanza universitaria después de la última Conferencia Mundial sobre la Educación Superior (UNESCO, 1998), es la pertinencia de la formación profesional en atención a las exigencias del mundo del trabajo y a la preparación del estudiantado para ser competente en un escenario laboral marcado por la mundialización y la internacionalización económica y social. Esto implica nuevas tendencias en el diseño 
de los planes de estudio, para orientarlos al desarrollo de capacidades generales y a la práctica, donde se privilegia el vínculo entre la teoría y la práctica y el aprendizaje interdisciplinario. En consecuencia:

Es evidente que las instituciones de educación superior y los gobiernos responsables, al reflejar los desafíos futuros del mundo del trabajo, necesitan mejor información sobre el empleo y el trabajo de los graduados, sobre el efecto de las disposiciones y condiciones del estudio en el futuro empleo y trabajo, y sobre las indicaciones relativas a los cambios tecnológicos, económicos y sociales a largo plazo. (UNESCO, 1998, p. 24)

Pese a la importancia de tener información precisa del sector empleador y del mundo del trabajo, existe el riesgo de omitir en este tipo de investigación a otros actores fundamentales: estudiantes, personal académico y autoridades universitarias. De no hacerse un análisis global, se puede caer en imprecisiones y llegar a conclusiones limitadas en relación con los planes de estudio, pues cada actor tiene sus propios intereses y necesidades. De ahí la importancia de convertir los estudios de seguimiento de la población graduada como un componente más de una investigación a profundidad del contexto laboral y profesional, así como de las tendencias de desarrollo nacional, regional y mundial. Estos estudios deben ser periódicos para dar seguimiento al desarrollo de la profesión y del plan de estudios.

En cuanto a la experiencia de la Escuela de Administración Educativa en la aplicación de estudios de seguimiento de la población graduada, interesa destacar que desde la década de 1990, sus procesos de renovación y cambio curricular han tomado como punto de partida este tipo de estudios y que hoy, en proceso de autoevaluación y acreditación de ambas licenciaturas, constituyen una fuente de información invaluable. Además, gracias a ellos, la Unidad Académica cuenta con un historial de su quehacer y, por ende, de la formación profesional en el campo, que contribuye a la teorización y desarrollo del conocimiento y tecnologías en la didáctica de la disciplina.

La información sobre el avance de la disciplina, las condiciones laborales y las demandas del ejercicio de la profesión, se debe obtener de la población graduada, del sector empleador y de la autoevaluación de la Unidad Académica, pues, sólo de esa forma se hace una verdadera renovación e innovación curricular. Ell valor de estos estudios es incuestionable, por lo que la Escuela de Administración Educativa no sólo se ha beneficiado con los resultados de estas investigaciones en el campo curricular, sino en relación con el desarrollo de la disciplina y los requerimientos y demandas sociales en relación con la práctica profesional. Ello contribuye a elevar la pertinencia, la calidad y la equidad de las carreras que ofrece la Unidad Académica y, por ende, de la formación universitaria pública, en un sector crítico para el desarrollo personal y social en el país: la gestión de la educación.

Con base en la experiencia acumulada y después de proyectos de investigación exito$\operatorname{sos}^{2}$, los estudios de seguimiento de graduados y la consulta al sector empleador se han consolidado en la Escuela de Administración Educativa y se hacen esfuerzos para que puedan llevarse a cabo cada cinco años, para asegurar una fuente de información que oriente la toma de decisiones en relación con el plan de estudios, los cursos, la investigación, la docencia y la acción social.

\section{Notas}

1 Véanse al respecto: Escuela de Administración Educativa. (1993). Planes de Estudio Licenciatura en Ciencias de la Educación con énfasis en: Administración Educativa y Administración de Programas de Educación no formal. UCR -Facultad de EducaciónEscuela de Administración Educativa.

2 Véanse al respecto la tesis de Licenciatura en Ciencias de la Educación con énfasis en Administración Educativa de Castillo, G., Mena, N., Espinoza, R. \& Orozco, V. (1991), y los informes finales de las investigaciones No. 241-A2-06 Descripción y análisis de la práctica de Administradores(as) Educativos(as) en la coyuntura actual (2004), y No. 241-A3-066 Estudio de 
seguimiento de graduados de la Licenciatura en Ciencias de la Educación con énfasis en Administración Educativa y en Administración de Programas de Educación no Formal (2004).

\section{Referencias bibliográficas}

Escuela de Administración Educativa. (1981). Fines, objetivos y funciones de la Escuela de Administración Educativa. Escuela de Administración Educativa, Universidad de Costa Rica: Mimeografiado.

Escuela de Administración Educativa. (1993). Planes de Estudio de la Licenciatura con énfasis en Administración Educativa $y$ en Administración de Programas de Educación no Formal. Escuela de Administración Educativa, Universidad de Costa Rica: Mimeografiado.

Glazman, R. (2001). Evaluación y exclusión en la enseñanza universitaria. Barcelona: Paidós.

Martínez, J. M. \& Gutiérrez, J. (1998). Métodos y técnicas cualitativas de investigación en Ciencias Sociales. Madrid: Síntesis.

Monereo, C. \& Pozo, J. I. (2003). La universidad ante la nueva cultura educativa. Madrid: Síntesis.
Pacheco, T. \& Días, A. (1997). La profesión. $\mathrm{Su}$ condición social e institucional. México, D.F.: Porrúa.

Pfeiffer, D. (1993). Formación universitaria y profesión. Chile: Universidad Austral de Chile.

Schomburg, H. (1995). Standard Instrument For Graduate And Employer Surveys. Germany: Eschborn And Kassel.

Schomburg, H. (2003). Manual para estudios de seguimiento de la población graduada. Alemania: Universidad de Kassel.

Tünnermann, C. (1999). Educación superior de cara al siglo XXI. San José, Costa Rica: Mirambell.

Vicenç, Benedicto, Ferrer, Virginia \& Ferreres, Vicente. (1995). La formación universitaria a debate. Barcelona: Universitat de Barcelona.

UNESCO. (1998, 5-9 de Octubre). Conferencia Mundial sobre la Educación Superior. Debate temático: Las exigencias del mundo del trabajo. Conferencia presentada en, Conferencia mundial sobre la Educación Superior en el siglo XXI: Visión y acción. París: UNESCO. 